\title{
Filling the Boundary Layer Gap Improved NWP with Drone and Lidar Data
}

\author{
Adapted from "Improving \\ High-Impact Numerical Weather \\ Prediction With Lidar and \\ Drone Observations," by Daniel \\ Leuenberger (Federal Office of \\ Meteorology and Climatology, \\ MeteoSwiss), Alexander Haefele, \\ Nadja Omanovic, Martin Fengler, \\ Giovanni Martucci, Bertrand \\ Calpini, Oliver Fuhrer, and \\ Andrea Rossa, Published online \\ in BAMS, July 2020. For the full, \\ citable article, see DOI:10.1175 \\ /BAMS-D-19-0119.1.
}

igh-impact weather is often determined by physical processes in the planetary boundary layer (PBL). For example, the PBL temperature and moisture distributions determine to a large degree the occurrence of thunderstorms. Fog and low stratus are common in central Europe during fall and winter and have a high socioeconomic impact. Accurate thermodynamic structure of the PBL in initial conditions is essential for numerical forecasts of fog.

The World Meteorological Organization (WMO) guidance for NWP applications states that wind, temperature, and humidity profiles, particularly for the PBL, are "not adequately measured by current or planned systems," both for global and high-resolution, regional models. The current upper-air observing system has strengths and weaknesses. For example, while spaceborne sensors generally have very good horizontal and temporal coverage, they are unable to observe in the lowest few kilometers of the atmosphere due to surface effects and clouds. Radiosondes provide very high-quality and high-vertical-resolution profiles of temperature, humidity, and wind, but generally only twice per day. Commercial aircraft generally can deliver quality temperature and wind profiles through the Aircraft Meteorological 
Data Relay, but rarely humidity. Finally, wind profilers provide wind information and are one of the best established PBL profiler systems.

WMO's Observing Systems Capability Analysis and Review (OSCAR) requirement levels for observations are: threshold (minimum for useful observations), breakthrough (significant improvement of application, optimum cost-benefit) and goal (ideal, no further improvements necessary). While the above mentioned observation systems generally meet the breakthrough requirements for quality and vertical resolution, they are not able to meet the requirements for horizontal and temporal resolution for lower-tropospheric temperature and humidity. Consequently, there is a significant gap in today's operational observing system in terms of thermodynamic quantities and wind in the PBL, inhibiting detailed and reliable forecasts of high-impact weather. We believe Raman lidar and rotary-wing unmanned aerial vehicles (UAVs; also referred to as drones] can help close the gap.

\section{Raman lidar and Meteodrones}

Raman lidar for Meteorological Observations (RALMO) has been operated by MeteoSwiss

\section{Equipped with $* D$ an emergency rescue system, Meteodrone is allowed to fly in "beyond visual line of sight" conditions at up to $3 \mathbf{~ k m ~ A G L . ~}$}

\section{Schematic picture of the Raman lidar for Meteorological Observations (RALMO) with its main elements $\frac{*}{\nabla}$}

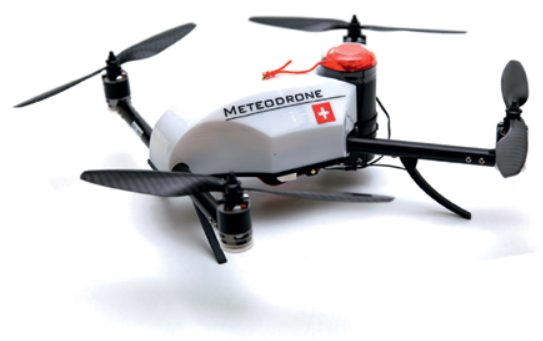

since 2006. It is fully automated, delivering a long-term average data availability of $50 \%$, and has been favorably validated against radiosondes. Profiles typically reach to $5 \mathrm{~km}$ AGL during the day and $12 \mathrm{~km}$ AGL during the night, with an integration time of $30 \mathrm{~min}$ and a vertical resolution on the order of $100 \mathrm{~m}$. Water vapor and temperature profiles are true 30-min averages and not instantaneous values, but still below the breakthrough requirement for high-resolution NWP, which is $60 \mathrm{~min}$. Raman lidars can be operated continuously, and profiles can be made available in near-real time. Deployment of a sufficiently dense operational network of Raman lidars might fill the observational gap in the lower troposphere.

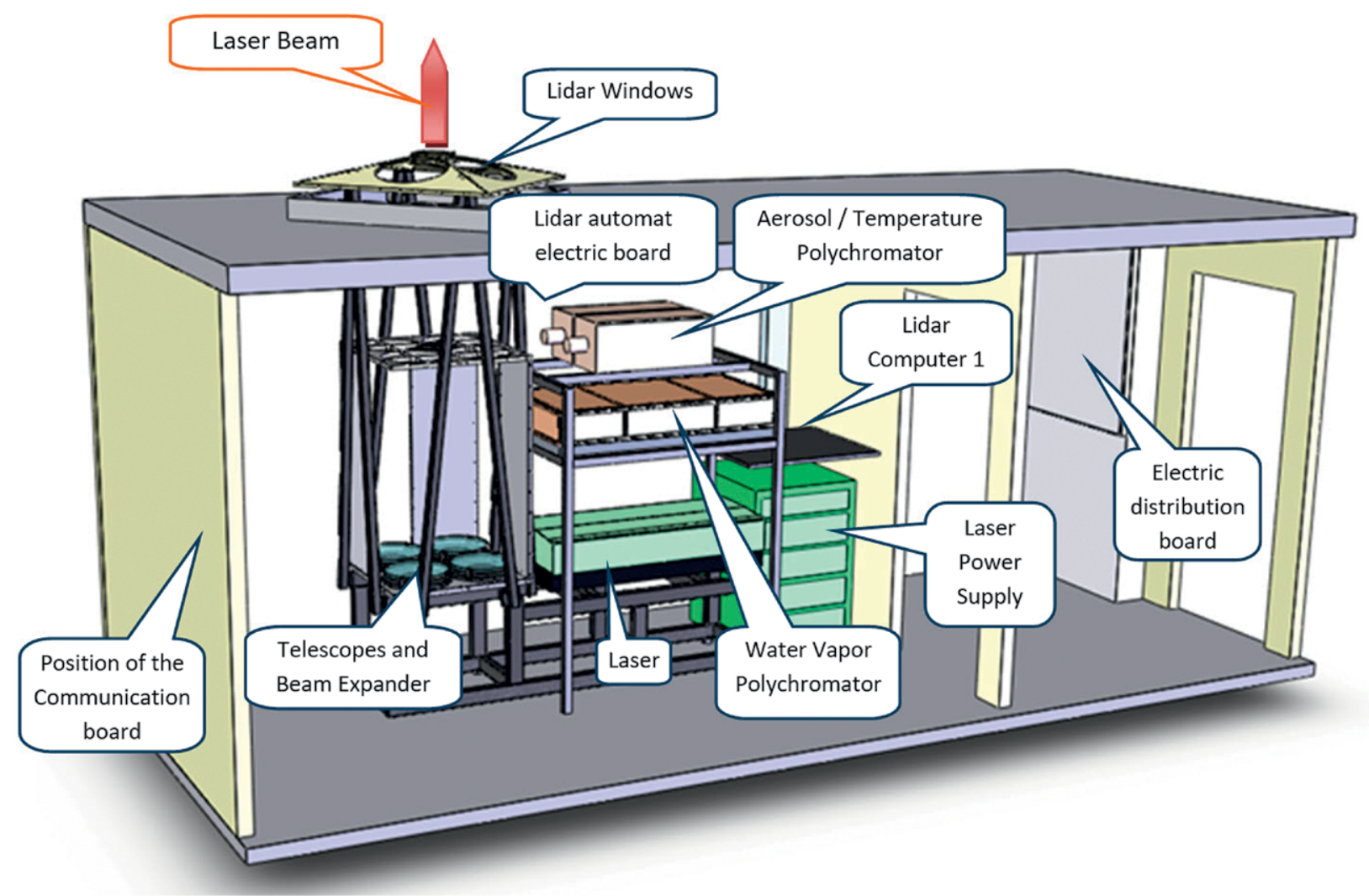




\section{Analysis}
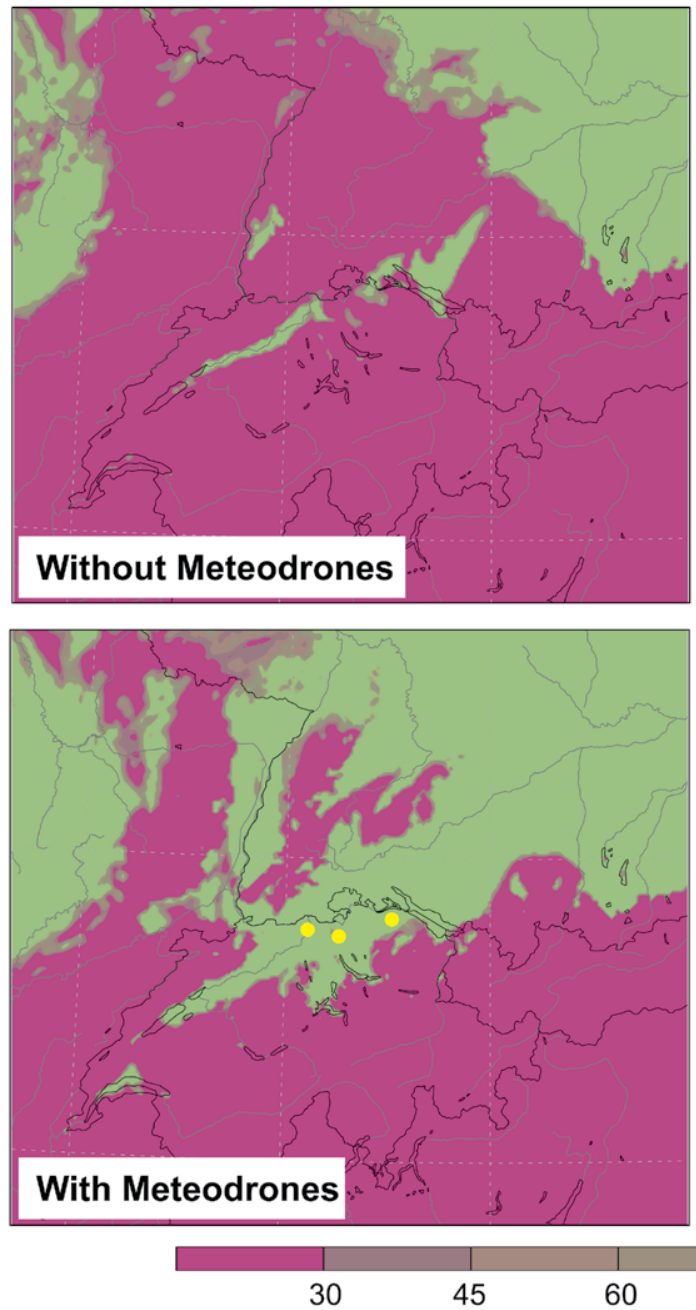

30

45

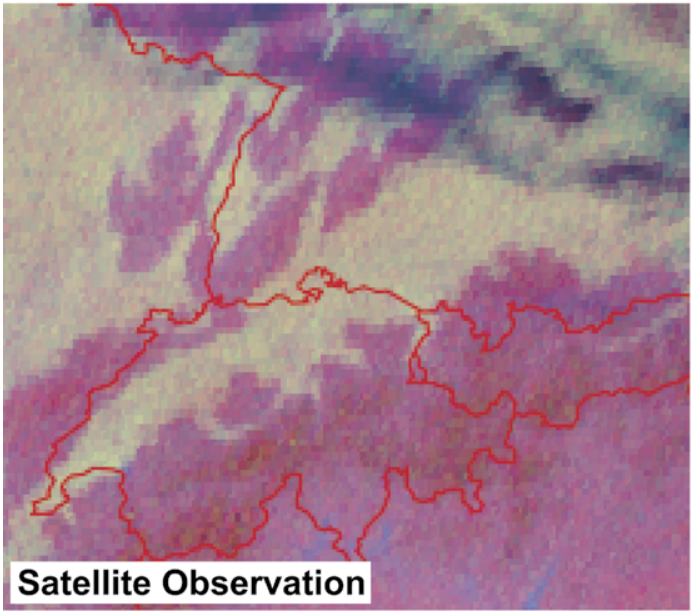

+6h Forecast
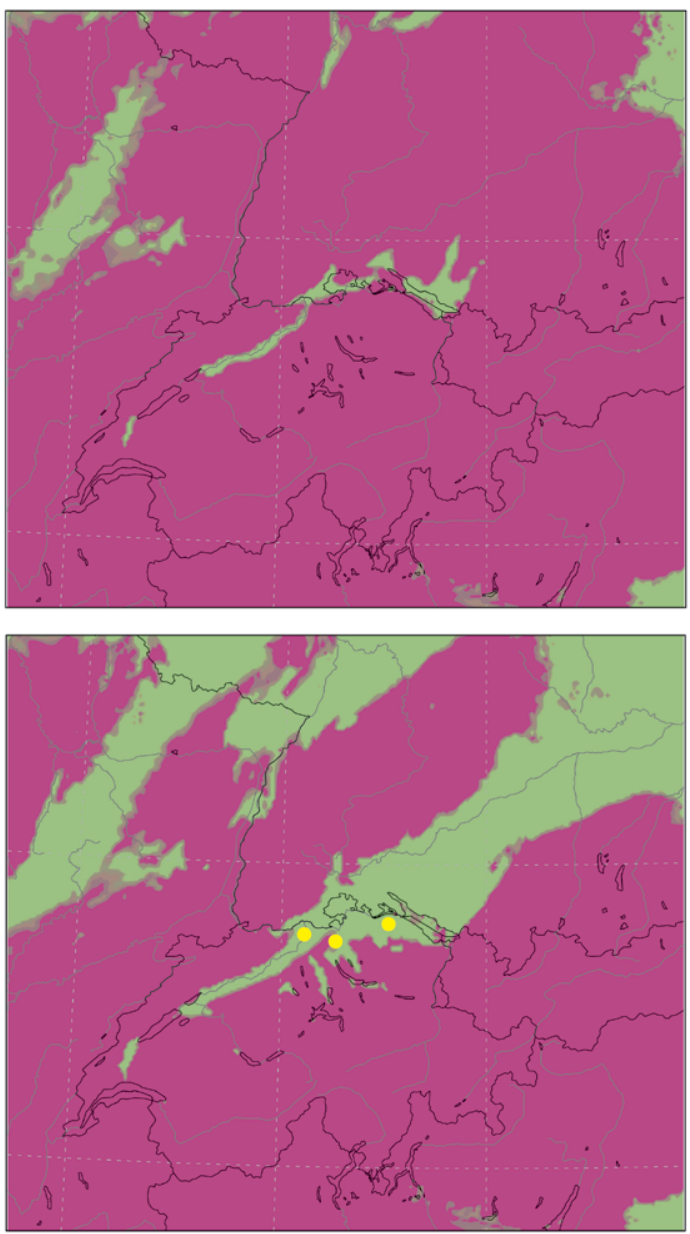

$\%$

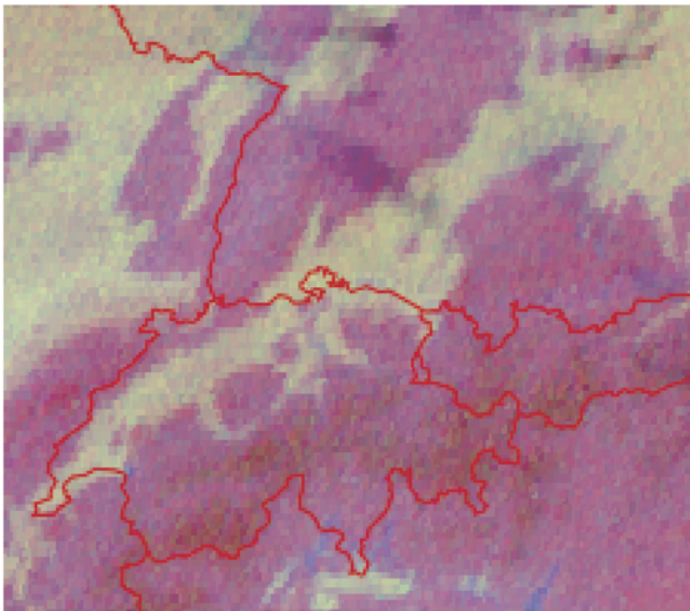

$\Delta$

* Fig. 8. Cloudiness at (left) 0000 UTC and (right) 0600 UTC 7 Dec 2017 from the COSMO ensemble mean (top) without and (middle) with the assimilation of Meteodrone observations. (left) Analysis time, and (right) $+6-\mathrm{h}$ forecast time. (bottom) Corresponding cloudiness as observed by satellite. Bright colors denote cloudy, purple colors cloud-free regions. The measurement locations of the Meteodrones are marked with yellow dots on the middle panels. The forecasts with Meteodrone observations assimilated shows a clearly improved cloud distribution at both, analysis and +6-h forecast time, compared to that without Meteodrone observations. 
The RALMO observations meet the breakthrough requirements for highresolution NWP.

Technical progress has made possible the use of UAVs in atmospheric research. Advantages of UAVs include near-continuous sampling, sampling across gradients, and no limitation to airspace directly above ships. UAVs have also provided useful information about the diurnal evolution of the PBL and mesoscale features above the PBL. Moreover, they have helped in the evaluation of finescale atmospheric models. Meteomatics's Meteodrones are reusable hexacopters with a span of $0.6 \mathrm{~m}$ that measure vertical profiles of the lower atmosphere at fixed locations, at high temporal frequency. Even though Meteodrone systems are very robust, these UAVs are vulnerable to icing and high winds. To solve the icing problem, a heating system for the propellers has been developed. The Meteodrone can sustain gusts up to $60 \mathrm{~km} \mathrm{~h}^{-1}$; above that, manual intervention is required. Like the lidar observations, the Meteodrone observations meet the breakthrough requirements for high-resolution NWP,
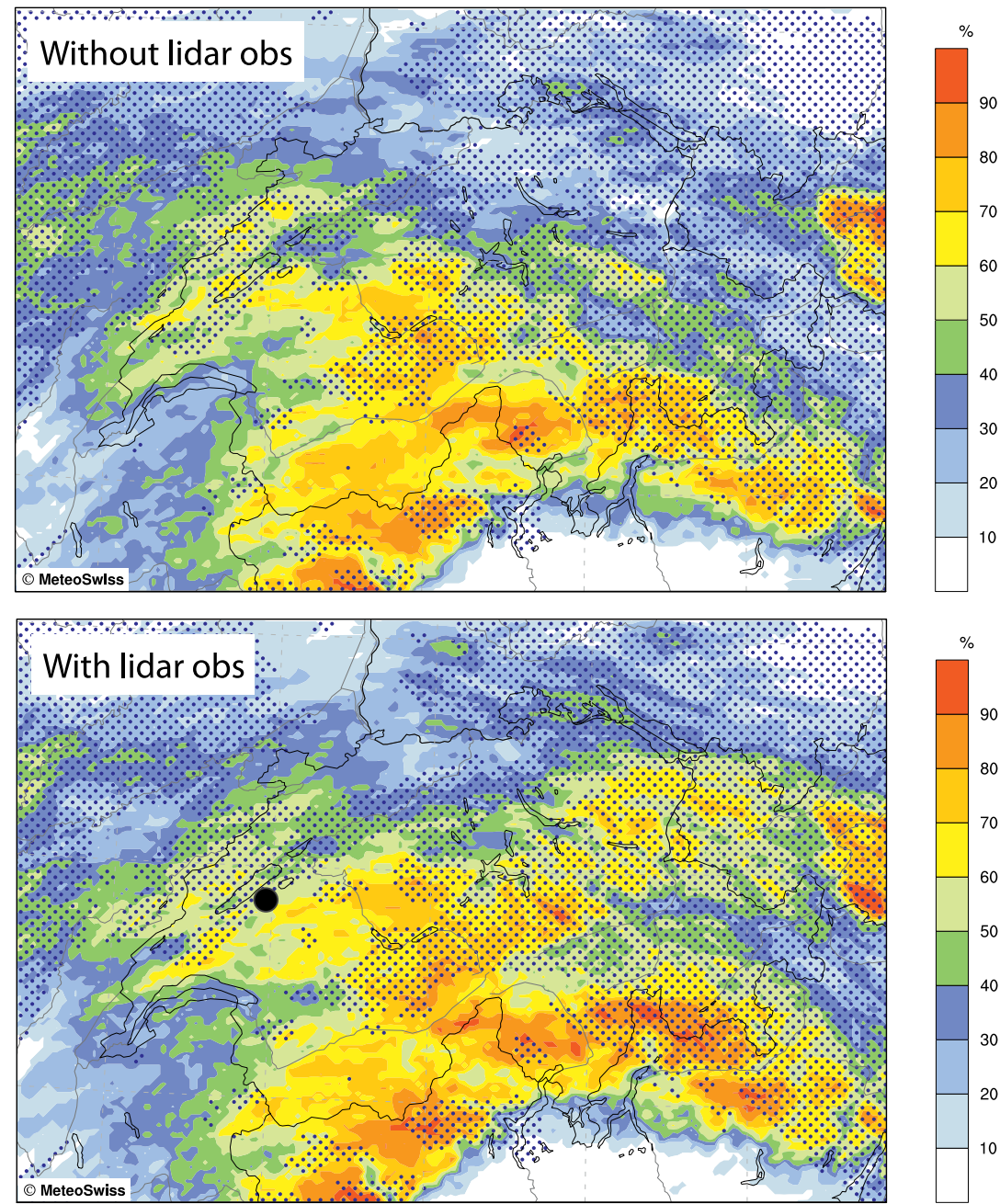
and have the potential to help fill the observational gap in the PBL.

\section{Impact on convective-scale NWP}

To investigate the impact of the novel observation systems on NWP, we conducted a series of assimilation experiments using the operational ensemble data assimilation and NWP system of MeteoSwiss for thunderstorms and fog.

On the afternoon of 8 July 2017, an organized convective system associated with a cold front crossed Switzerland from west to east, with precipitation totals up to $80 \mathrm{~mm}$. The experiments involved 24 hourly analysis cycles with and without the assimilation of RALMO observations, followed by a 24-h forecast. The forecast initialized with the analysis with RALMO observations shows higher probabilities of rain in eastern Switzerland (consistent with observations) than the forecast initialized without RALMO data. This precipitation forecast clearly benefited from assimilation of the additional

\section{$\Delta$} * Forecasted probability of the 24-h accumulated precipitation ending at 0000 UTC $9 \mathrm{Jul} 2017$ to exceed $1 \mathrm{~mm}$ $24 \mathrm{~h}^{-1}$ (color shading). Stippled shading denotes regions where the observed precip-

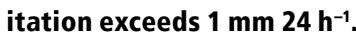
Upper (lower) panel shows the forecast with (without) lidar observations included. A good forecast is characterized by a high probability in the stippled areas. The measurement location of Payerne is marked with a black dot in the lower panel. The forecast with lidar measurements assimilated has a better agreement of high probabilities and observed precipitation than the one without lidar data.
RALMO observations. Another case study using assimilated RALMO data (in August 2017) exhibited a similar improvement in the convective precipitation forecast.

To assess the impact of the Meteodrones observations on NWP analy-ses and fog forecasts, we chose seven case studies. On 7 December 2017, a high-pressure system meant weak synoptic-scale forcing over Switzerland. In such situations, the Swiss Plateau is often completely covered with fog. With standard observations, the model substantially underestimated the fog extent. Conversely, the assimilation of the Meteodrones observations cooled and moistened the PBL and allowed fog to form in a region that closely resembles the verifying satellite image. The positive 
impact of the Meteodrone observations persisted for at least $6 \mathrm{~h}$.

In two other weak forcing events (6 December 2017 and 28 February 2018), the impact on cloudiness was clearly positive, while in three cases with stronger synoptic-scale forcing, the impact was neutral. No negative impact has been found in the seven investigated cases.

\section{Potential for a global observing system}

Permanent upper-air networks in Switzerland, Japan, and the United States continuously provide critical parameters in the PBL and lower troposphere for NWP applications. Raman lidars and drones have not been used in these networks up to now, mainly because of insufficient automation and reliability.

UAVs are still a very young technology and flight regulations differ from country to country, hindering the establishment of a broad network. However, steps have been taken toward standardization of these regulations across Europe. Another challenge for a UAV network is full automation.

The design of a future network of lidars and drones depends on a variety of factors, including meeting OSCAR requirements for vertical and temporal resolution as well as horizontal spacing of the devices in a network. Results of one study suggest that even a network with a station spacing well above the OSCAR threshold requirement on horizontal resolution can benefit NWP. More research is needed to find optimal measurement strategies and network densities for lidar and Meteodrones.

Given the developments described above, we believe that lidar and drones will become standard equipment for temperature and humidity profiling over the coming decade. While lidar and drones are likely to be used initially to enhance observations in sensitive areas for high-resolution NWP and nowcasting, they might eventually become an important component of the WMO Integrated Global Observing System. We expect that continuous assimilation of lidar and drone observations into a high-resolution NWP model will result in a more realistic PBL structure of temperature and humidity.

\section{$\equiv$ METADATA}

BAMS: What would you like readers to learn from this article?

\section{Daniel Leuenberger (Federal Office of Meteorology and Climatology, MeteoSwiss):}

There are exciting new technologies in measuring atmospheric quantities that are largely underexplored in the context of numerical weather prediction. These new observations can make the difference between fog and no fog in the model forecasts and thus be of large economic and societal benefit for the public.

BAMS: How did you become interested in this topic?

DL: The collaboration between modelers and observation specialists is very fruitful and of mutual benefit. I got to know the lidar observations when intensifying collaboration with our observation colleagues at MeteoSwiss in Payerne and the Meteodrones when meeting Martin Fengler of Meteomatics. Both observation systems fascinated me from the beginning, and we thought the model could profit therefrom.

BAMS: What surprised you the most about your findings?

DL: Honestly, I did not think that the impact of the new observations in the model would be so large!

BAMS: What was the biggest challenge you encountered in this work?

DL: An NWP model is a complex beast, and getting new observations into it is highly nontrivial. With the help of colleagues at Deutscher Wetterdienst, we managed to get every bit to where it belongs.

BAMS: What's next?

DL: We are working hard to assimilate the new observations routinely in our model and make it therefore even more useful. 